\title{
Stokes-Einstein diffusion of colloids in nematics
}

Frédéric Mondiot, Jean-Christophe Loudet, Olivier Mondain-Monval, Patrick Snabre Centre de Recherche Paul Pascal, Université de Bordeaux \&3 CNRS, 33600 Pessac, France

\author{
Alexandre Vilquin, Alois Würger \\ Laboratoire Ondes et Matière d'Aquitaine, Université de Bordeaux 85 CNRS, 33405 Talence, France
}

\begin{abstract}
We report the first experimental observation of anisotropic diffusion of polystyrene particles immersed in a lyotropic liquid crystal with two different anchoring conditions. Diffusion is shown to obey the Stokes-Einstein law for particle diameters ranging from $190 \mathrm{~nm}$ up to $2 \mu \mathrm{m}$. In the case of prolate micelles, the beads diffuse four times faster along the director than in perpendicular directions, $D_{\|} / D_{\perp} \approx 4$. In the theory part we present a perturbative approach to the Leslie-Ericksen equations and relate the diffusion coefficients to the Miesovicz viscosity parameters $\eta_{i}$. We provide explicit formulae for the cases of uniform director field and planar anchoring conditions which are then discussed in view of the data. As a general rule, we find that the inequalities $\eta_{b}<\eta_{a}<\eta_{c}$, satisfied by various liquid crystals of rodlike molecules, imply $D_{\|}>D_{\perp}$.
\end{abstract}

PACS numbers: 05.40.Jc; 82.70.Dd; 83.80.Xz

Dispersions of colloids in nematic liquid crystals (NLCs) show singular properties, that are related to the anisotropy of the nematic phase and to the anchoring of the nematogens on the particle surface 1 1-4. The colloid imposes on the neighboring LC molecules an orientation that locally breaks the uniform nematic alignment and gives rise to elastic interactions. In order to satisfy the global boundary conditions, each inclusion is accompanied by topological defects that determine the long-range deformation field and govern colloidal pattern formation [5, 11.

A Brownian particle in a NLC thus drags a nematic deformation along its random trajectory, and its diffusion behavior constitutes a sensitive probe to the local order parameter and surface anchoring. In an isotropic medium, the Stokes-Einstein coefficient $D=k_{B} T / 3 \pi \eta d$ is given by the particle diameter $d$ and the scalar viscosity $\eta$. A more complex situation arises in a nematic, where viscosity is a tensor quantity and where the director and velocity fields exert forces on each other [12, 13]. Thus the viscous stress of a diffusing particle on the surrounding fluid, is not the same for motion parallel and perpendicular to the director, resulting in two coefficients $D_{\|}$and $D_{\perp}$.

So far anisotropic colloidal diffusion has been studied in thermotropic NLCs, made of rod-like organic molecules the anchoring of which is determined by the surface chemistry 14,18. Rather generally, diffusion turns out to be faster along the director, with a ratio $D_{\|} / D_{\perp}$ smaller than 2. A recent study on silica beads dispersed in nematic $5 \mathrm{CB}$ with normal anchoring conditions [18], reported that the diffusion coefficients $D_{\|}$and $D_{\perp}$ of large particles show the size dependence $D \propto 1 / d$ expected from the Stokes-Einstein relation, yet surprisingly saturate for smaller particles at an effective hydrodynamic diameter of about $300 \mathrm{~nm}$. The role of surface chemistry, which controls the anchoring of the nematogens, was put forward in an attempt to rationalize the observations.

Since boundary conditions are of paramount impor-

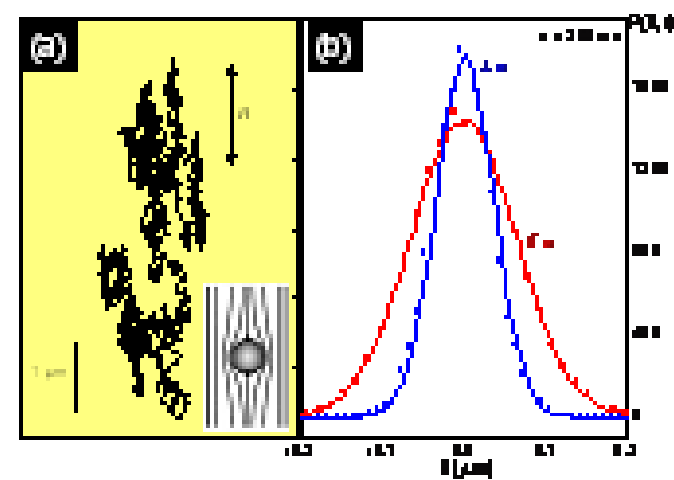

FIG. 1: (a) Brownian trajectory of a 190nm-diameter fluorescent PS particle, consisting of 2800 time steps of $0.3 \mathrm{~s}$. Diffusion is faster parallel to the director (double arrow). Inset: Schematic of the director field distortions around a sphere in planar anchoring conditions. The black dots symbolize "boojum" defects [3. (b) Histogram of the measured particle displacement $\delta$ parallel and perpendicular to the director during a time $\tau=0.3 \mathrm{~s}$, as obtained from a sample of 20,000 trajectory steps. The solid lines are Gaussian fits.

tance in nematic colloids, we found it worthy to investigate particle mobility in lyotropic liquid crystals for which various anchoring conditions are easily achieved without altering the surface chemistry [19]. The latter does indeed influence the particle diffusion coefficients as shown in Ref. [17. Lyotropic LCs are water-based surfactant mixtures, and in such systems, anchoring conditions depend critically on the shape of the surfactant micelles (nematogens). The latter can be tuned, through tiny changes of surfactant concentrations, from rodlike (Calamitic Nematics, NC phase) to disklike (Discotic Nematics, ND phase) [20, 21]. And for entropic reasons 


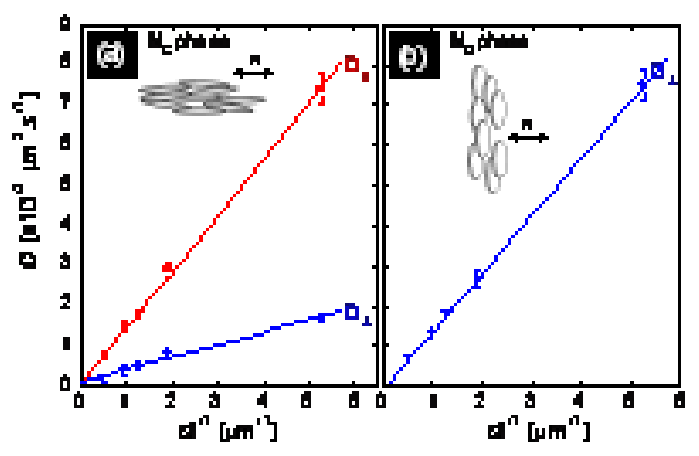

FIG. 2: Stokes-Einstein evolution of the diffusion coefficients $D_{\|}$(along the nematic director) and $D_{\perp}$ (perpendicular to the nematic director) as a function of the particle diameter. The ratio $D_{\|} / D_{\perp}$ is about equal to 4 .

[22], spontaneous planar (NC) and normal (ND) anchoring conditions can be achieved at constant surface chemistry (and consequently constant anchoring strength), in contrast with the thermotropic case where varying the anchoring conditions requires a change in the particles surface chemistry.

In this Letter, we report on both experimental and theoretical work on anisotropic diffusion. We present data for particles with diameters $d$ ranging from $190 \mathrm{~nm}$ to $1.9 \mu \mathrm{m}$, both in planar and normal anchoring conditions of the lyotropic LC at the particle surface. To the best of our knowledge, these are the first data obtained in lyotropic systems and in both anchoring conditions. In the theory part, we develop an original perturbative approach which provides the effective viscosities to linear order in terms of the Leslie coefficients. The obtained expressions enable a direct comparison with the experimental relevant quantities and can account for the observed diffusion anisotropy.

The two NLC phases used are the NC and the ND phases of the water/decanol/sodium dodecyl sulfate lyotropic system, which can be obtained at very close experimental concentrations (NC 71/24.5/4.5 \%; ND $73 / 23.5 / 3.5 \%$ ) [20, 21]. In the NC phase, the surfactant molecules form nanometer-sized rodlike (prolate) micelles (with long and short axes of $9 \mathrm{~nm}$ and $3.5 \mathrm{~nm}[23]$ ), which, in order to minimize their excluded volume, show planar anchoring at the surface of the dispersed polystyrene spheres (PS). In the ND phase, and for similar reasons, the disklike (oblate) micelles (with diameter $8 \mathrm{~nm}$ and thickness $3.5 \mathrm{~nm}$ [23]) anchor normally at the surface (with the disk normal perpendicular to the surface) [19]. Unlike colloidal suspensions in thermotropic NLCs, this dispersion does not require surface functionalization.

We used classical and fluorescence optical microscopy combined with standard video tracking routines 24 to probe the Brownian diffusion in lyotropic phases. A typical trajectory of a $190 \mathrm{~nm}$-diameter particle derived from 2,800 snapshots is shown in Fig. (1a) for the NC case. Its elongation along the nematic director $\mathbf{n}$ indicates that diffusion is faster in this direction. In the histogram of Fig. (1c), we plot the displacements parallel and perpendicular to $\mathbf{n}$, for a total of 20,000 trajectory steps. The probability that the particle moves a distance $\delta$ in time $\tau, P(\delta, \tau)$, is very well fitted by a Gaussian distribution; its standard deviation is related to the diffusion coefficient through $\overline{\delta^{2}}-\bar{\delta}^{2}=2 D \tau$ 25. In Fig. (2a), we plot $D_{\|}$and $D_{\perp}$ as a function of the inverse particle radius in the $\mathrm{NC}$ phase whereas Fig. (2b) displays the results for the ND phase. However, due to experimental limitations [26], only $D_{\perp}$ could be determined in the latter case. The straight lines confirm the linear dependence of the Stokes-Einstein relation whatever the anchoring conditions. The friction coefficients are different for motion parallel and perpendicular to $\mathbf{n}$ and the large anisotropy ratio $D_{\|} / D_{\perp} \approx 4$ indicates a strong nematohydrodynamic coupling in the LC matrix. Note also the very close values measured for $D_{\perp}$ in the ND phase and $D_{\|}$in the NC phase. Our results then differ considerably from the measurements of [18] in a themotropic NLC, where the diffusion coefficients become constant for particles smaller than about $300 \mathrm{~nm}$, with $D_{\|} / D_{\perp} \approx 1.6$.

In the remainder of this paper, we study how the diffusion anisotropy arises from the viscous properties of a NLC. The fluctuation-dissipation theorem relates the friction coefficient to the velocity $u=F / 3 \pi \eta d$ of a spherical particle driven by an external force $F$. Thus calculating the Rayleigh function $\Psi=F u$ provides an effective viscosity in the form $\Psi=3 \pi \eta d u^{2}$, which takes two values $\eta_{\|}$and $\eta_{\perp}$ for motion parallel and perpendicular to the director.

The friction coefficients are calculated from the LeslieEricksen equations of nemato-hydrodynamics for $|\mathbf{n}|=1$ and an incompressible fluid. Energy dissipation occurs through two channels [12, 13,

$$
\Psi=\int d V \psi, \quad \psi=\sigma: \mathbf{A}+\mathbf{h} \cdot \mathbf{N},
$$

where $\mathbf{A}$ and $\mathbf{N}$ are thermodynamic fluxes, and $\sigma$ and $\mathbf{h}$ the corresponding forces. The rate of strain tensor

$$
A_{i j}=\frac{1}{2}\left(\partial_{i} v_{j}+\partial_{j} v_{i}\right)
$$

is given by the symmetrized derivatives of the flow $\mathbf{v}(\mathbf{r})$ in the vicinity of the particle moving at velocity $\mathbf{u}$. The vector quantity

$$
\mathbf{N}=((\mathbf{v}-\mathbf{u}) \cdot \nabla) \mathbf{n}-\omega \times \mathbf{n},
$$

with the curl $\omega=\frac{1}{2} \nabla \times \mathbf{v}$, expresses the rate of change of the director with respect to the background fluid. The conjugate forces, that is the viscous stress tensor $\sigma$ and the molecular field $\mathbf{h}$, are linear functions of the components of $\mathbf{A}$ and $\mathbf{N}$ [12, 13. Inserting their steady-state 
TABLE I: Effective viscosities for zero anchoring (uniform director). The middle column is obtained from Eqs. (6) and (7) with the Leslie coefficients of 5CB and MBBA [12, 13, the last one is derived from Stark's numerical calculations [3].

\begin{tabular}{|l|c|c|c|}
\hline \multicolumn{2}{|l|}{ Uniform director } & present work & numerically exact \\
\hline $5 \mathrm{CB}$ & $\eta_{\|}(\mathrm{P})$ & 0.429 & 0.381 \\
\hline & $\eta_{\perp}(\mathrm{P})$ & 0.724 & 0.754 \\
\hline $\mathrm{MBBA}$ & $\eta_{\|}(\mathrm{P})$ & 0.412 & 0.380 \\
\hline & $\eta_{\perp}(\mathrm{P})$ & 0.650 & 0.684 \\
\hline
\end{tabular}

expressions in (1) one has

$$
\begin{aligned}
\psi=\alpha_{1} & (\mathbf{n} \cdot \mathbf{A} \cdot \mathbf{n})^{2}+\left(\alpha_{3}-\alpha_{2}\right) \mathbf{N}^{2} \\
& +\left(\alpha_{3}+\alpha_{2}+\alpha_{6}-\alpha_{5}\right) \mathbf{n} \cdot \mathbf{A} \cdot \mathbf{N} \\
& +\alpha_{4} \mathbf{A}: \mathbf{A}+\left(\alpha_{5}+\alpha_{6}\right) \mathbf{n} \cdot \mathbf{A} \cdot \mathbf{A} \cdot \mathbf{n} .
\end{aligned}
$$

Parodi's relation $\alpha_{3}+\alpha_{2}=\alpha_{6}-\alpha_{5}$ reduces the viscosity tensor to five independent Leslie coefficients $\alpha_{i}$. The various scalar products result in an intricate dependence on the relative orientation of the macroscopic director $\mathbf{n}_{0}$ and the particle velocity $\mathbf{u}$.

In the absence of nematic ordering, $\mathbf{n}=0$, the power density reduces to $\psi=\alpha_{4} \sum_{i j} A_{i j}^{2}$. The tensor 2 is readily calculated from the velocity field of a spherical particle moving in an isotropic liquid,

$$
\mathbf{v}=\left(\frac{3 a}{4 r}(1+\hat{\mathbf{r}} \hat{\mathbf{r}})+\frac{a^{3}}{4 r^{3}}(1-3 \hat{\mathbf{r}} \hat{\mathbf{r}})\right) \cdot \mathbf{u}
$$

where $\hat{\mathbf{r}}=\mathbf{r} / r$. The resulting Rayleigh function $\Psi_{0}=$ $\frac{3}{2} \pi d \alpha_{4} u^{2}$ defines the isotropic viscosity $\eta_{0}=\frac{1}{2} \alpha_{4}$. In a NLC, however, the velocity and director fields depend on each other through the equations for $\sigma$ and $\mathbf{h}$. Then $\Psi$ is a complicated function of the Leslie coefficients, and it is not possible to single out the dissipation due to a given term. Though the problem can be solved with considerable numerical effort [1, 3, 27 30 , the resulting numbers for the effective viscosities give no physical insight in the underlying mechanism.

The present work relies on two approximations. First, we evaluate (2) and (3) with the above velocity field $\mathbf{v}(\mathbf{r})$ of a particle in an isotropic liquid. Formally this amounts to linearize $\psi$ with respect to the $a_{i}$. Second we use a simple parameterization for the director $\mathbf{n}(\mathbf{r})$ which is independent of the velocity field and which depends on the particle size through the reduced distance $d / r$ only; in other words, the director has no intrinsic length scale. With these assumptions, the Rayleigh function becomes linear in the $\alpha_{i}$ and in the particle size $d$.

Uniform director (UD). We start with the case where the particle surface does not affect the liquid crystal order parameter. Then the director is constant, $\mathbf{n}=\mathbf{n}_{0}$, and with the explicit form of the tensor $\partial_{i} v_{j}$ [31, the dissipation function can be calculated in closed form. It turns out convenient to rewrite the Leslie parameters $\alpha_{2}, \ldots, \alpha_{6}$ in terms of the Miesovicz viscosities $\eta_{a}, \eta_{b}, \eta_{c}$ given in
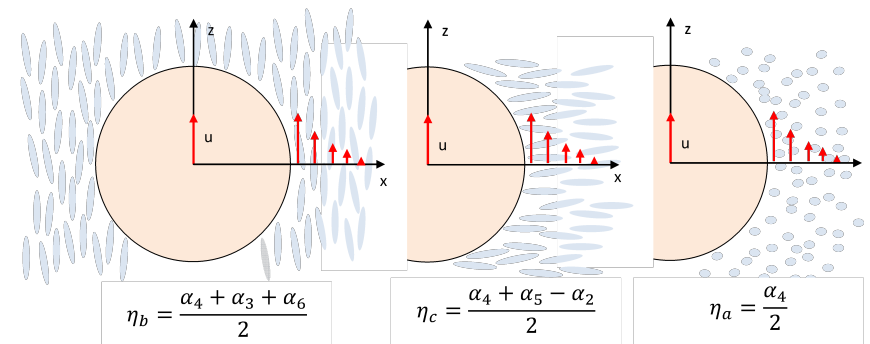

FIG. 3: Schematic view of a colloidal particle in a liquid crystal. The particle moves along the $z$-axis; the shear in the plane $z=0$ is indicated by the decay of the fluid velocity field. In the left panel the director is parallel to the particle velocity, with an effective shear viscosity $\eta_{b}$. The remainder shows the perpendicular case $\mathbf{n}_{0}=\mathbf{e}_{x}$; in the middle a view of the $x$-z-plane with $\eta_{c}$, at right the $y$-z-plane with $\eta_{a}$. The Miesovicz viscosities $\eta_{a}, \eta_{b}, \eta_{c}$ are expressed in terms of the Leslie parameters.

Fig. 3; the effective viscosity for a particle moving along the nematic order, $\mathbf{u} \| \mathbf{n}_{0}$, then reads

$$
\eta_{\|}^{U D}=\frac{8 \alpha_{1}}{70}+\frac{4 \eta_{b}+\eta_{c}}{5} .
$$

Similarly, we find for the perpendicular case $\mathbf{u} \perp \mathbf{n}_{0}$

$$
\eta_{\perp}^{U D}=\frac{3 \alpha_{1}}{70}+\frac{5 \eta_{a}+\eta_{b}+4 \eta_{c}}{10} .
$$

It is noteworthy that the five independent viscosities of (4) reduce to three or four terms. In Table I we compare our formulae with Stark's numerical calculations for the liquid crystals $5 \mathrm{CB}$ and MBBA [3, and find that the numbers differ by hardly $10 \%$. Though it slightly underestimates the viscosity anisotropy, the linearization approximation is therefore quantitatively correct.

Because of the small weight of the first term in (6) and (7), the anisotropy arises mainly from the Miesovicz viscosities. Its physical origin is illustrated in Fig. 3 for a particle moving in the vertical direction. The pole regions being of minor importance, we focus on the shear flow in the plane $z=0$, where the viscous stress simplifies to the in-plane derivatives of the vertical velocity component $\partial v_{z} / \partial x$ and $\partial v_{z} / \partial y$. The left panel shows the parallel case, and the middle and right panels the perpendicular one, with the corresponding shear viscosities. This qualitative picture is confirmed by the large weight of $\eta_{b}$ in the parallel viscosity (6), and of $\eta_{a}$ and $\eta_{c}$ in (7). Data for common NLCs made of rodlike molecules suggest that $\alpha_{1}$ is small; more importantly, they satisfy the inequalities $\eta_{b}<\eta_{a}<\eta_{c}$ [13] and thus imply $\eta_{\|}<\eta_{\perp}$, which is in line with our results.

Planar anchoring (PA). A finite surface energy deforms the nematic order parameter in the vicinity of the colloidal particle. Here we consider the case of planar anchoring, which is illustrated in the inset of Fig. 1a. As the distance from the particle increases, $\mathbf{n}$ varies smoothly toward the constant $\mathbf{n}_{0}$. Even for the simplified oneconstant elastic energy, there is no general solution for 
TABLE II: Coefficients of the effective viscosity (9) for a spherical particle with planar anchoring (PA), moving parallel or perpendicular to the director. The measured values are obtained by fitting the straight lines in Fig. (2a) with $D=k_{B} T / 3 \pi \eta d$.

\begin{tabular}{|c||c|c|c|c||c|}
\hline PA & $C_{1}$ & $C_{b}$ & $C_{c}$ & $C_{d}$ & measured \\
\hline \hline$\eta_{\|}$ & 0.08 & 1.02 & 0.40 & -0.48 & 0.31 Pa.s \\
\hline$\eta_{\perp}$ & 0.04 & 0.24 & 0.41 & -0.15 & 1.24 Pa.s \\
\hline
\end{tabular}

the spatially varying order parameter [3]. It is conveniently parameterized by

$$
\mathbf{n}=\mathbf{n}_{0} \cos \Theta-\mathbf{n}_{\perp} \sin \Theta
$$

where $\mathbf{n}_{\perp}$ is a radial vector perpendicular to $\mathbf{n}_{0}$. Because of its rotational symmetry, the director is determined by a single function $\Theta(\mathbf{r})$, which decays as $1 / r^{3}$ at large distances. Here we use the ansatz of Lubensky et al. 8, which for planar anchoring results in $\Theta=\sum_{k=1}^{\infty}\left[\sin \left(2 k \theta_{n}\right) / k\right](d / 2 r)^{1+2 k}$, where $\theta_{n}$ is the polar angle with respect to $\mathbf{n}_{0}$, that is $\cos \theta_{n}=\hat{\mathbf{r}} \cdot \mathbf{n}_{0}$.

With the director (8) and the velocity (5), the dissipation function (1) is calculated numerically for both parallel and perpendicular alignement; it yields to the following viscosities

$$
\eta=C_{1} \alpha_{1}+\left(1-C_{b}-C_{c}\right) \eta_{a}+C_{b} \eta_{b}+C_{c} \eta_{c}+C_{d} \eta_{d}
$$

where we have defined a fifth independent parameter $\eta_{d}=\frac{1}{2}\left(\alpha_{6}-\alpha_{3}\right)=\frac{1}{2}\left(\alpha_{5}+\alpha_{2}\right)$. From the numerical coefficients in Table II it is clear that the viscosity anisotropy arises essentially from $C_{b}$ and $C_{d}$. Since $C_{d}=0$ for a uniform director, finite values of $C_{d}$ reflect distortions due to anchoring. In the following, we discard the first term because of the small coefficient $C_{1}$. (Moreover, $\alpha_{1}$ is also small for several thermotropic NLCs [12, 13]).

Comparison with experiment. From the straight lines in Fig. (2a) (NC phase, planar anchoring) and the Stokes-Einstein relation, we deduce the experimental values $\eta_{\|}=0.31$ Pa.s and $\eta_{\perp}=1.24$ Pa.s. Using a platecone rheometer, the zero shear effective viscosity was found to be $\eta_{S} \simeq 0.4$ Pa.s at $T=25^{\circ} \mathrm{C}$. Though these numbers are not sufficient to determine all Leslie parameters, they present several noteworthy constraints. In view of the coefficients listed in Table II and Eq. (9), we deduce $\Delta \eta=\eta_{\|}-\eta_{\perp} \simeq\left(C_{b}^{\|}-C_{b}^{\perp}\right)\left(\eta_{b}-\eta_{a}\right)+$
$\left(C_{d}^{\|}-C_{d}^{\perp}\right) \eta_{d}$. From experiments, $\Delta \eta<0$, which therefore implies $\eta_{b}<\eta_{a}$ and suggests that $\eta_{d}$ is small or positive. The zero shear effective viscosity is given by $\eta_{S}=m_{a} \eta_{a}+m_{b} \eta_{b}+m_{c} \eta_{c}$, with $m_{i}=\frac{1}{3}$ in a polycrystalline sample. Because of the planar anchoring conditions on the confining surfaces of the rheometer, we expect a smaller weight for $\eta_{c}$; in addition, shear-induced alignment would reduce $m_{a}$. Indeed, we find that the three equations for $\eta_{\|}, \eta_{\perp}$, and $\eta_{S}$ have solutions only if $m_{a, c}<0.15$ and $m_{b}>0.7$, and strongly suggest $\eta_{a}<\eta_{c}$. These inequalities are satisfied by the Miesovicz parameters that are required to fit our data: For example, setting $\eta_{d}=0$ and $m_{a, c}=0.05$, the measured viscosities are met with $\eta_{b}=0.27$ Pa.s, $\eta_{a}=1.46$ Pa.s, $\eta_{c}=1.61$ Pa.s. This discussion qualitatively agrees with that of $\eta_{\perp}$ measured for planar anchoring in the thermotropic NLC 5CB [17].

Finally, we close with a remark on diffusion in discotic nematics (ND phase) where the anchoring is normal and not planar anymore. As aforesaid, the data for the perpendicular coefficient $D_{\perp}$ in Fig. (2b) are almost identical to those for $D_{\|}$in the NC phase. As discussed above, the left panel of Fig. 3 implies $\eta_{\|} \sim \eta_{b}$ for prolate micelles; a similar argument for oblate ones suggests $\eta_{\perp} \sim \eta_{c}$. Consequently, the important point is that, in the ND phase, one expects the Miesovicz viscosities to satisfy $\eta_{c}<\eta_{a}<\eta_{b}$. Thus, it does not come as a surprise that $\eta_{\perp}(\mathrm{ND})$ and $\eta_{\|}(\mathrm{NC})$ take close values. This argument implies moreover that diffusion in the ND phase should be faster perpendicular to the director, i.e. $D_{\|}<D_{\perp}[26$.

In summary, we have investigated diffusion in lyotropic LCs with two different anchoring conditions. Our measurements confirm Stokes friction $D \propto 1 / d$ in both cases, unlike a previous study on thermotropic NLCs 18. In the NC phase, we found an unusually large viscosity ratio $\eta_{\perp} / \eta_{\|} \approx 4$ which can be accounted for thanks to our perturbative theoretical approach. Our analysis imposes $\eta_{b}<\eta_{a}<\eta_{c}$ on the Miesovicz parameters in the NC phase (which is usually the case 32]), and suggests $\eta_{c}<\eta_{a}<\eta_{b}$ with $D_{\|}<D_{\perp}$ in the ND phase. As a short-term follow-up work, we will evaluate Eq. (9) for the case of normal anchoring and an independent measurement of the Miesovicz viscosities together with the additionnal parameter $\eta_{d}$ would be most desirable.

We acknowledge financial support from the French National Research Agency under grant \# ANR-07-JCJC0023 and the Conseil Régional d'Aquitaine.
[1] R. W. Ruhwandl and E. M. Terentjev, Phys. Rev. E 54, 5204 (1996).

[2] P. Poulin, H. Stark, T.C. Lubensky, and D. A. Weitz, Science 275, 1770 (1997).

[3] H. Stark, Phys. Rep. 351, 387 (2001).

[4] Y. Bai and N.L. Abbott, Langmuir 27, 5719 (2011).

[5] R. W. Ruhwandl and E. M. Terentjev, Phys. Rev. E 55,
2958 (1997).

[6] H. Stark, Eur. Phys. J. B 10, 311 (1999).

[7] P. Poulin and D. A. Weitz, Phys. Rev. E 57, 626 (1998).

[8] T.C. Lubensky, D. Pettey, N. Currier, H. Stark, Phys. Rev. E 57, 610 (1998).

[9] J. C. Loudet, P. Barois, and P. Poulin, Nature 407, 611 (2000). 
[10] I. Muševič et al., Science 313, 954 (2006).

[11] G.M. Koenig Jr., J.J. de Pablo, and N.L. Abbott, Langmuir 25, 13318 (2009).

[12] P.-G. de Gennes and J. Prost, The Physics of Liquid Crystals, 2nd ed. (Clarendon Press Oxford, 1993).

[13] P. Oswald and P. Pieranski, Nematic and Cholesteric Liquid Crystals (Taylor \& Francis, 2005).

[14] J.-C. Loudet, P. Hanusse, P. Poulin, Science 306, 1525 (2004).

[15] I.I. Smalyukh, A.V. Kachynski, A.N. Kuzmin, and P.N. Prasad, PNAS 103, 18048 (2006).

[16] K. Takahashi, M. Ichikawa and Y. Kimura, J. Phys. Cond. Matt. 20, 075106 (2008).

[17] G. M. Koenig Jr., J. J. de Pablo, R. Ong, A. D. Cortes, J. A. Moreno-Razo, and N. L. Abbott, Nano Letters 9, 2794 (2009).

[18] M. Škarabot and I. Muševič, Soft Matter 6, 5476 (2010).

[19] P. Poulin, N. Francès, and O. Mondain-Monval, Phys. Rev. E 59, 4384 (1999).

[20] L. Q. Amaral, M.E. Marcondes Helene, J. Phys. Chem. 92, 6094 (1988).

[21] P. O. Quist, B. Halle, and I. Furó, J. Chem. Phys. 96, 3875 (1992).

[22] A. Poniewierski A. and R. Holyst, Phys. Rev. A 38, 3721 (1988).

[23] A. Nesrullajev, Mater. Chem. Phys. 123, 546 (2010).
[24] J. C. Crocker and D. G. Grier, J. Colloid Interface Sci. 179, 298 (1996).

[25] P. M. Chaikin, T. C. Lubensky, Principles of Condensed Matter Physics (Cambridge Univ. Press, Cambridge, 1995).

[26] For the following reason, we could not measure this anisotropy. In the ND phase, the director spontaneously orients perpendicular to the sample glass slides along the $z$-direction. Since the photographs are recorded in the $x y$-plane, only $D_{\perp}$ could be probed with our imaging setup. More fancy methods, such as video holographic microscopy [see for example S.-H. Lee et al., Opt. Express 15, 18275 (2007)] should enable measurements of $D_{\|}$in this phase.

[27] J. Fukuda, H. Stark, M. Yoneya, and H. Yokoyama, J. Phys. Condens. Matter 16, S1957 (2004).

[28] C. Zhou, P. Yue, and J. J. Feng, Langmuir 24, 3099 (2008).

[29] S. Carlotto and A. Polimeno, J. Chem. Phys. 128, 154505 (2008).

[30] J. A. Moreno-Razo et al., Soft Matter 7, 6828 (2011).

[31] L. D. Landau and E. M. Lifshitz, Fluid Mechanics (Elsevier, New York, 1987).

[32] M. Simões and S. M. Domiciano, Phys. Rev. E 68, 011705 (2003). 